\title{
Present radiotelescopes: single-dish, interferometer and telescope networks
}

\author{
José Antonio López Fernández ${ }^{1}$ \\ Instituto Geográfico Nacional (IGN) \\ Centro Astronómico de Yebes \\ Yebes (Guadalajara) Spain \\ E-mail: ja.lopez@oan.es
}

Large fully steerable radiotelescopes are extensively used for radioastronomy and geodetic applications in the microwave and millimeter wave range. Single dish, connected interferometry and Very Long Baseline Interferometry (VLBI) observational techniques involve the construction of big collecting areas with high precision drive systems, high precision surfaces, ultra-high sensitivities and complex electronic processes.

In this document we intend to review some of the main aspects in the design and manufacturing of big size and high precision surface radiotelescopes. Different engineering topics will be discussed and some examples based on our experience with the construction of the 40 meter IGN radiotelescope will illustrate them.

Finally, a list of the main radiotelescopes and arrays all over the world with their main characteristics will be detailed.

2nd MCCT-SKADS Training School Radio Astronomy: fundamentals and the new instruments

Sigüenza (Spain)

August $26^{\text {th }}-$ September $4^{\text {th }} 2008$

1 speaker 


\section{Introduction}

The radiotelescope construction involves many fields of engineering. Civil works, optical, radiofrequency, electronics, software and mechanical engineering need to be coordinated in order to obtain a big collecting high precision surface with high accurate pointing. A big effort on mechanical engineering is needed for the design of the steel structure that supports the reflector, with ratios between surface rms accuracy and antenna diameter in the order of $10^{-6}$. A high precision mechanical with very accurate servomechanism design are also needed to obtain pointing accuracy in the order of arcseconds with structures having deadweights of nearly 500 tons.

Good electromagnetics (optical and radiofrequency) and cryogenics skillness is also needed. The design of the optical geometry of the system and of the feeds that illuminate the main reflector plays a crucial role in the antenna performance. Highly sensitive receivers involve the development of cryostats and low temperature instrumentation.

Software engineering is also very much involved for servo control and remote control sessions. Finally data low frequency electronics and data processing fulfill this always growing list of engineering tasks.

\section{Optical design}

A diversity of axially symmetric antenna configurations are widely used, being the most common the Cassegrain configuration. A hyperbolidal subreflector is interposed between the focal point of the paraboloidal reflector and provides a constant path length for the rays from the feed (at the system focus) to the aperture plane. This or some designs based on this, are very appropriate configurations because their high efficiency, low aberrations, low noise system temperature performance and easy accessibility to electronic receiver equipment.

The IGN 40 meters radiotelescope (see Fig. 1) is a Nasmyth-Cassegrain system, a modification of the basic Cassegrain design [1], with high equivalent ratio, $F / D=7.9$, high magnification, $\mathrm{M}=21$, and a Cassegrain focus placed 11 meters from the vertex of the parabola. The beam passing through the main reflector vertex hole encounters the Nasmyth mirror, M3, and it is reflected 90 degrees from the optical axis. M3 mirror moves in elevation compensating the antenna movement. The electronic equipment is then free from the elevation and azimuth movements of the antenna and remains fixed.

Two flat mirrors, M4 and M4', are placed both sides of M3 and redirects the beam parallel to the optical axis to the receivers. The M3 mirror can be rotated $180^{\circ}$, choosing one of the mirrors, fig. 2 . This improves the number of receivers in the receiver cabin. 


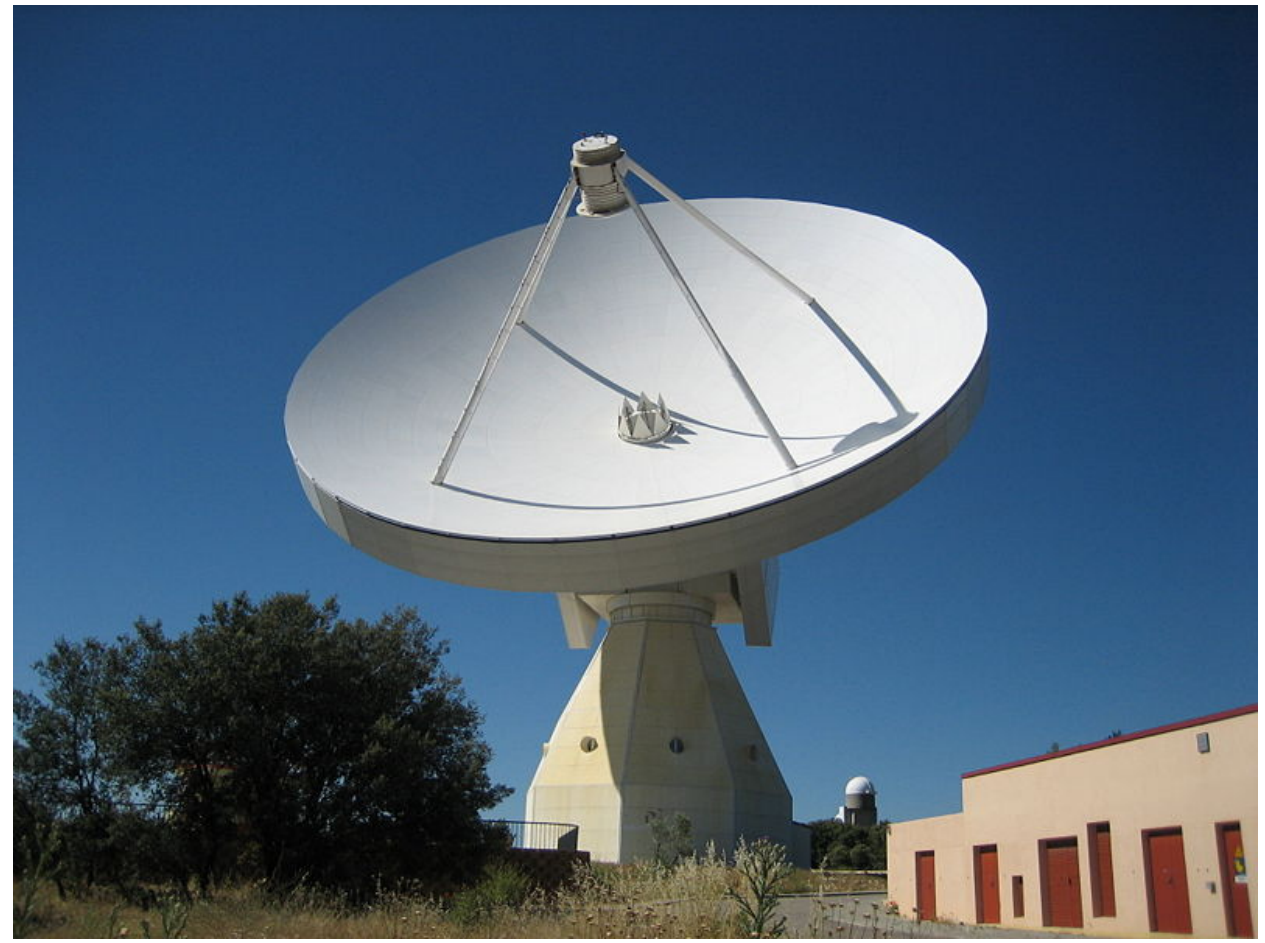

Figure 1: The IGN 40 meter radiotelescope.

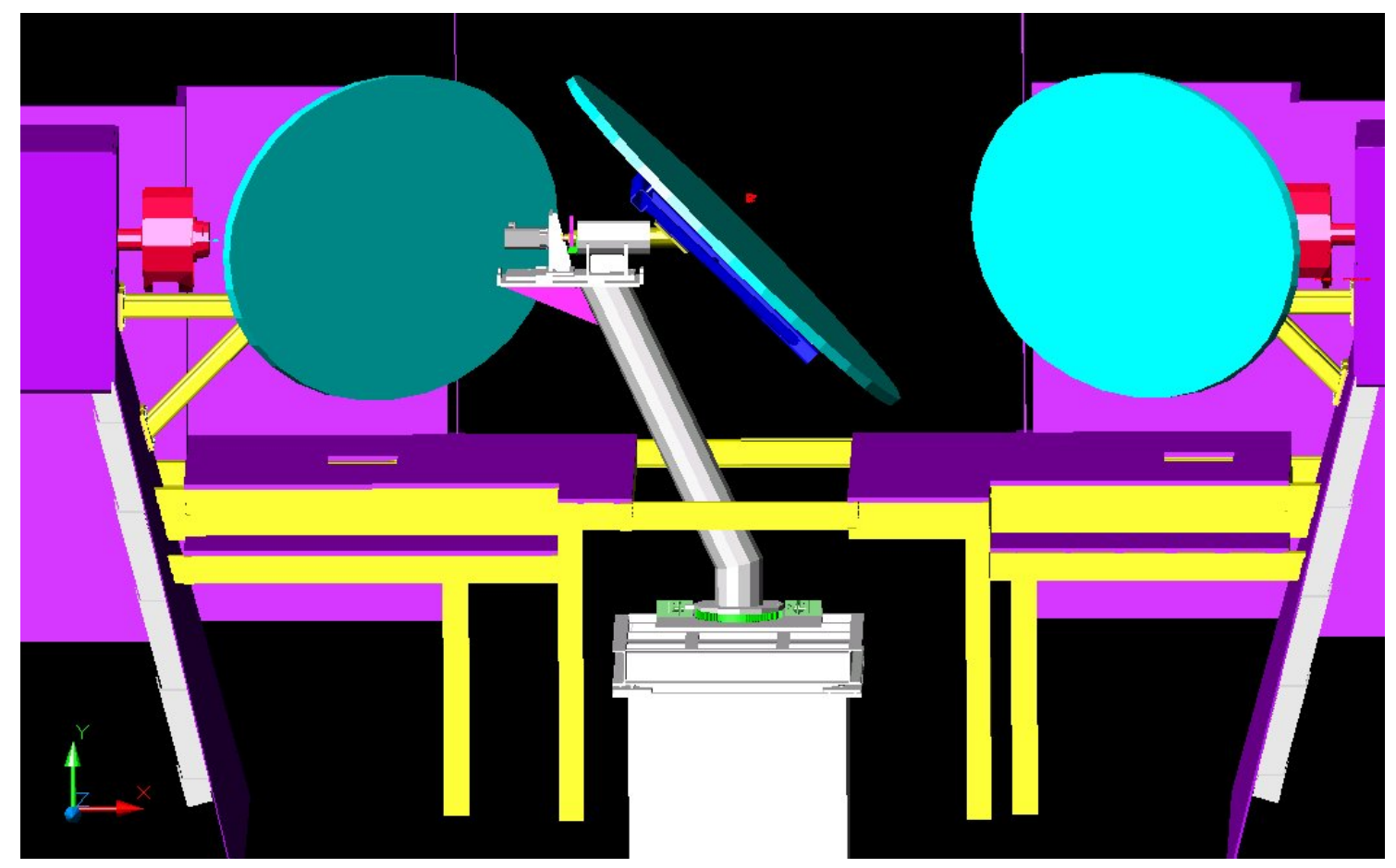

Figure 2: Nasmyth mirror configuration of the 40 meter IGN radiotelescope. 
Once the geometrical design is fixed, the RF synthesis and analysis is made using methods such as Physical Optics (PO) or Ray-tracing. The first is the method usually employed in the analysis of the radiation characteristics of multireflector systems in the microwave and millimeter range. It is based on the calculation of currents on each reflector and by far it is the most accurate but at the expense of computation time. GRASP [2] is a well known commercial software based on this technique. At the other end of the spectrum is ray tracing which ignores diffraction effects entirely.

The design of the feeds or the reflectors placed on the focal zone can be achieved with Gaussian beam techniques or quasioptics [3]. Gaussian beam analysis is a very powerful, fast and easy to understand tool that can be accurate enough to design complicated multireflector systems. It uses an appropriate set of expansion functions to model the field between reflectors. The field is then computed at the next reflector by using the expansion functions and their coefficients. Gaussian beam analysis is fully appropriate to radiotelescopes with high F/D ratios and Gaussian feeds. The most Gaussian feed is the corrugated feed horn [4]. Most of the radiastronomy feeds are based on this design.

In practice most of the designs start with a feaseability study with ray tracing, are designed with gaussian beam optics and are finally tested with PO. This is the case of the M4' branch of the 40 meter radiotelescope, fig. 3. A complex system with up to seven receivers, focusing mirrors, lenses and flat and dichroic mirrors has been design using quasioptics and has been tested using GRASP.

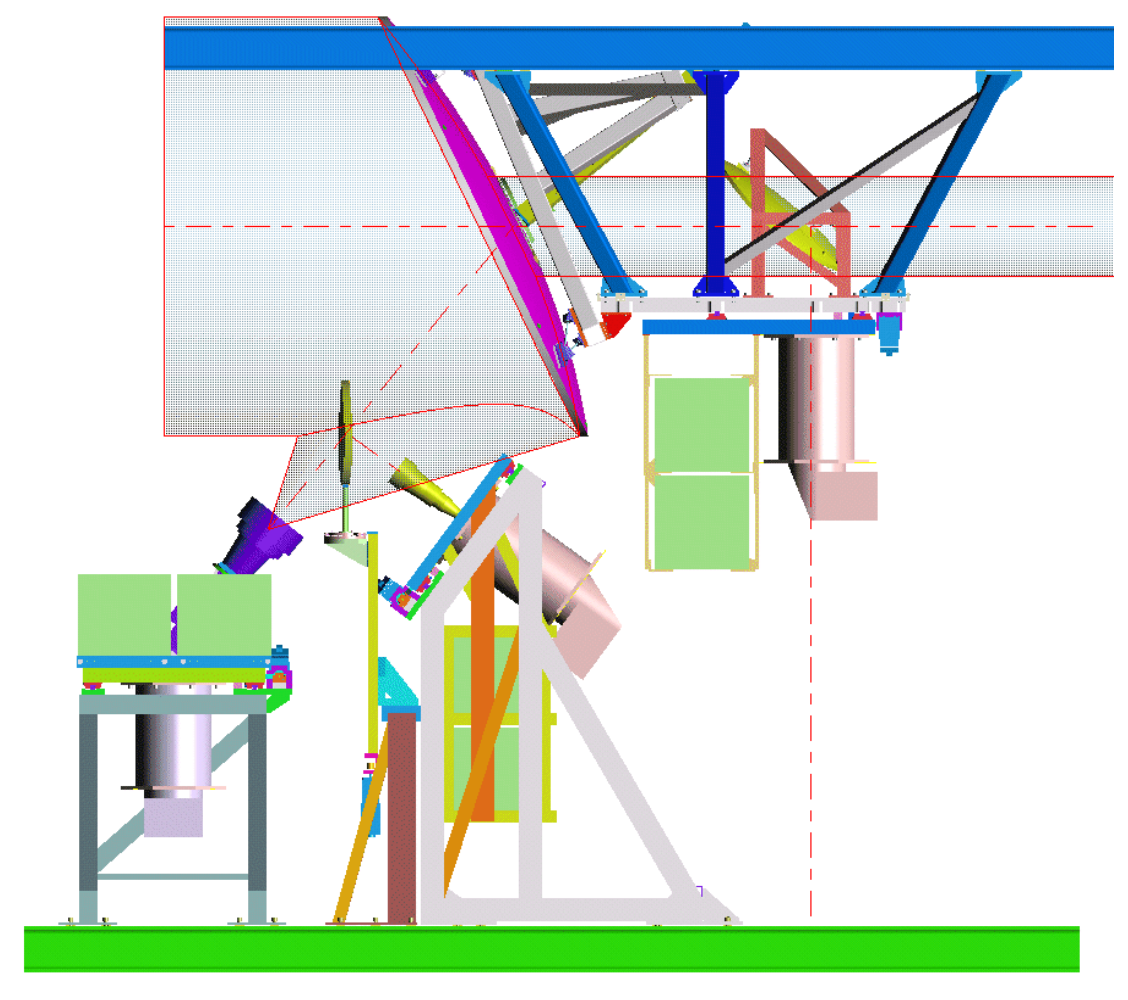

Figure 3: M4’ optics. 


\section{Mechanical design}

The basic specification in the mechanical design of a radiotelescope is that the antenna must be pointed in the required direction within the desired accuracy, and that the reflector must achieve the required surface accuracy. Typically, the pointing accuracy is around $10 \%$ of the half power beamwidth and the surface accuracy around the $5 \%$ of the wavelength. For the 40 meter radiotelescope this means 5" for the pointing and 150 microns rms for the surface accuracy.

The antenna structural design is usually achieved by fine element analysis (FEM) [5] and covers the following tasks: determine the structural elements and construct a model, analyze the strength of the structure, calculate deformations, analyze the natural frequencies and calculate the surface error pointing.

This analysis is specified under three sets of wind conditions: operation, steerable and survival winds. For the 40 meter radiotelescope four wind condition ranges have been specified: operation below $10 \mathrm{~m} / \mathrm{s}$, degraded operation below $15 \mathrm{~m} / \mathrm{s}$, steerable to stow position below 20 $\mathrm{m} / \mathrm{s}$, maximum steerable to stow position at $33 \mathrm{~m} / \mathrm{s}$ and survival wind at $50 \mathrm{~m} / \mathrm{s}$.

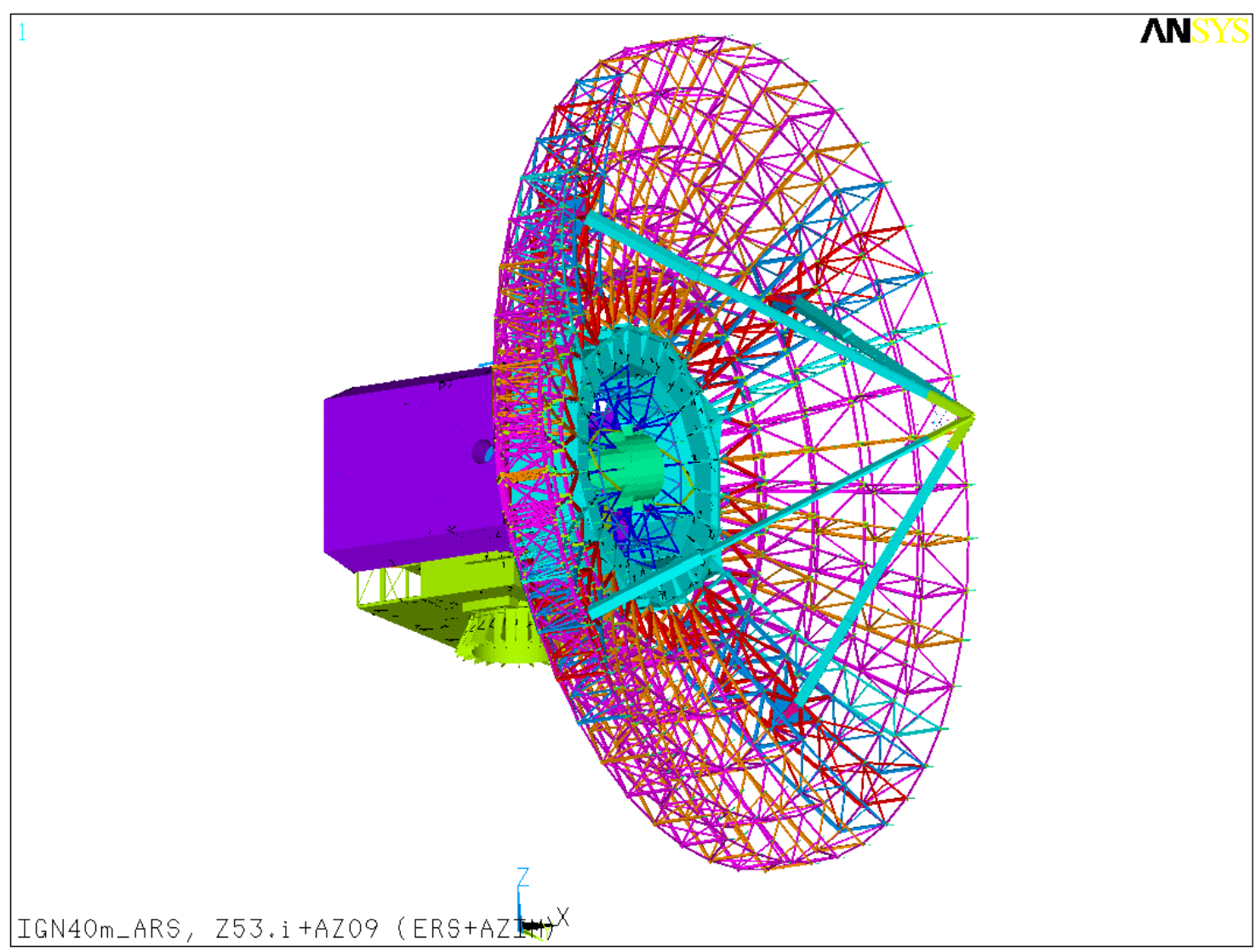

Figure 4: Mechanical FEM model for the 40 meter IGN radiotelescope. 
One of the most difficult problems in the structural design of large antennas is the prevention of reflector surface deformation due to deadweight and wind loading. In design large antennas better surface accuracy is obtained by designing the structure so that the reflector surface deforms from one paraboloid to another when rotated in elevation. This is termed a homologous deformation [6].

A homologous structure is designed for optimum performance under light wind conditions and without temperature effects. Therefore when very high surface accuracy is required special precautions must be taken to maintain an uniform temperature distribution. The rear side of the backstructure must be covered with thermal insulation panels and fans must be provided for fixed air circulation in the space between the reflector panels and the insulation panels.

Another important issue is related to the natural frequencies. The natural frequencies are the main factor that determine the response to variable loads such as non static winds and earthquakes. We aim to achieve high natural frequencies to reduce the effects of time varying loads. However for larger antennas the mass increases more rapidly than the stiffness and high frequencies cannot be achieved easily. A large antenna is designed so that the natural frequencies are greater than $2 \mathrm{~Hz}$, as the 40 meter radiotelescope. Having low resonant frequencies also reduces the complexity of the servo loop, mainly because the band pass of the servo must be lower than the lowest resonant frequency of the antenna to prevent violent instability [7].

\section{Mechanical description}

The antenna mechanical system is composed of three main units: reflector assembly, antenna mount, and drive system.

Fully steerable antennas have been often designed in the elevation over azimuth axis configuration which is the most economical. For microwave mounts, large diameter bearings are used to form the axis about which the azimuth axis rotates. For larger antennas, azimuth rotation is provided by a circular track with wheels at the base of a large frame called the alidade. These are very rigid mounts where track diameter and support frame structures can be very large, maintaining wind effect low and resonant frequencies high. However there is a high cost of leveling the rail and for the foundations. Nowadays, the bearing fabrication is competitive and presents the only drawback of the hypothetical replacement if damaged.

The design of the 40 meters radiotelescope is based on the concept of a turning head antenna with both axis elevation and azimuth carried out with roller bearings and geared drives. The telescope, made of steel, is supported by a conical concrete tower with several operations and auxiliary rooms inside it. The concrete tower and the azimuth rotating structure and the reflector rear side are protected against insolation and ice accumulation by an outside cladding. 
All parts of the telescope are mainly welded together and fabricated with a high grade accuracy that allows the welding to be done with a minimum distortion and to remain within the flatness and straightness tolerances and specified dimensions. The steel structure comprises the azimuth bearing supporting structure including apron and central tube bearing, the fork platform including the receiver cabin central, the access facilities and the reflector trusswork including the tetrapod.

The fork consists of a platform of hollow box type welded steel construction and the two fork arms, the inner space of the platform is used as an operating room in which the azimuth and elevation drives are arranged. The access facilities comprise the central tube with the spiral staircase including the guidance at the anchor ring, the annular landing above the cable twister, the staircase between central fork room and upper level of the receiver cabin and all safety rails.

The drive system is a feedback control system whose forward elements are the servo amplifier, the encoders and the drive assembly. The servo amplifier receives the angle error signal from the error detector as a result of the difference between the commanded position and the reading of the encoders. The amplified angle-error signal supplies power to the drive assembly that moves the antenna mount in the direction where the angle error signal is reduced to zero.

The drive assembly, fig. 5, is composed of motors and reduction gears. Electric drive dc motors are used, they have excellent speed versus torque characteristics and can be controlled over a wide speed range. The reduction gear consists of a gear train normally based on planetary gear train. The reduction gears are accompanied by backslash, precision antennas are provided with duplicate motors and reduction gears to which opposite bias torques are applied to reduce backslash. Each axis of the 40 meter radiotelescope is equipped of four drives (motor and gearbox) working opposite in order to improve this backslash performance.

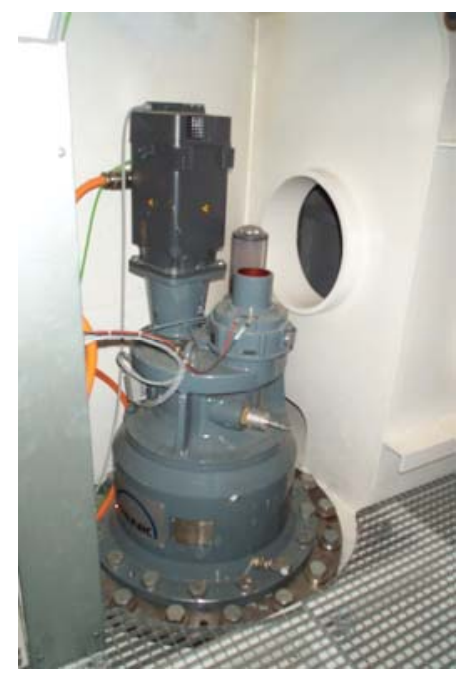

Figure 5: Drive assembly. 


\section{The reflective surface}

The reflective surface is made of a number of pie panels arranged in concentrical rows. The reflector panels can be of mainly two types: single skin or sandwich type panel. A single skin type consists of a thin aluminium plate, shaped to have the required surface, with stiffeners attached to the rear side of the plate. The most common way is the stretched-formed sheet. In this process the edges of the sheet are pulled over a curved mold. A Z section framework is then glued and riveted to the curved sheet metal. When the assembled panel is released from the tooling it has a parabolic shape.

The single skin panel is lower in cost, but also comparatively low in stiffness. Very good surface accuracy is difficult to achieve (not better than 50 microns rms) because the production errors of the skin are added to the assembly error. This technology was used in the 40 meters radiotelescope panels and the accuracy of most of the panels is below 65 microns rms.

A sandwich panel consists of a lightweight material with skins attached to the front and rear surfaces. The core usually is composed of a honeycomb or foam material, whereas the skin may be aluminum or metallized plastic such as glass fiber reinforced or carbon fiber reinforced plastic. This technology was used for the IRAM and ALMA radiotelescopes [8, 9], having better accuracy (near 20 micron rms), high stiffness and lightweight but more expensive manufacturing.

Surface accuracy of panels are tested using different technologies. The most common is direct measuring and comparison on a template by gauges, such as the sweep boom template measurement. New methods as photogrammetry offers higher measurement accuracy and easy of measurements. Furthermore, this method allows the measurement at different environmental conditions (temperature, gravity, wind...). This method was used for the characterization of the 40 meter panels, fig. 6 .

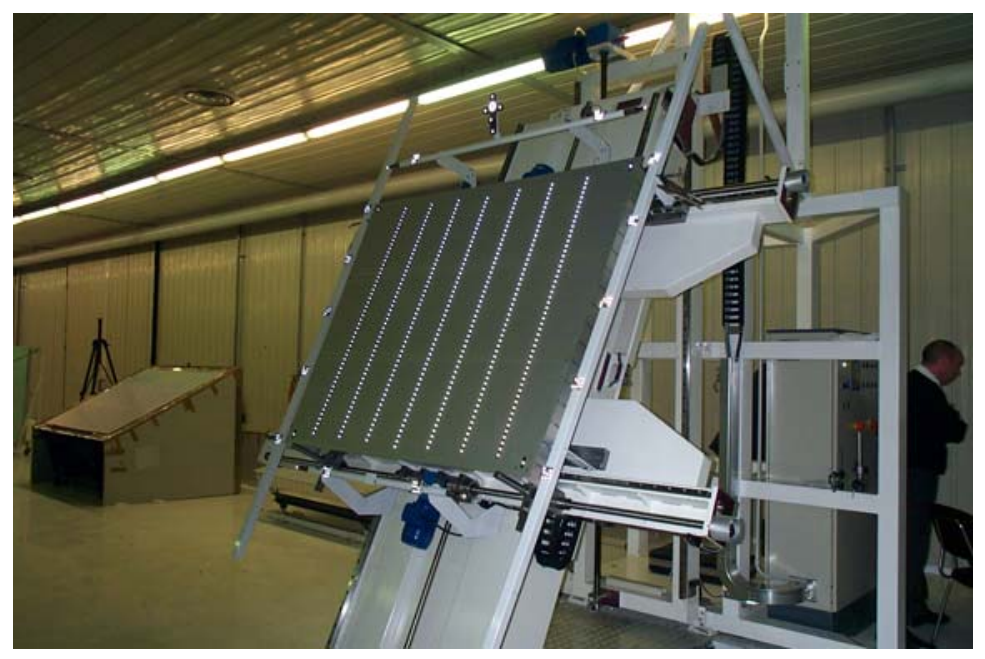

Figure 6: Panel test bench for testing at different elevations using photogrammetry. 


\section{Telescope construction}

The telescope construction starts with the workshop assembly of the steel parts and the pedestal (the concrete tower) construction on site. During the workshop phase some important assemblies need to be performed. First, the azimuth bearing should be integrated with its lower and upper supporting rings and its proper movement have to be checked. A common practice and a very important step is the trial assembly of the trusswork. All the truss parts should be assembled in the workshop in order to verify that they fit before are sent to the site. Other trial assemblies for the azimuth fork, the cantilevers with the gear rims or the tetrapod are appropriate practice. Finally, the mounting of the elevation bearings with the journals and axle housings is then performed; previously the bearing clearance is adjusted.

Corrosion protection is an important issue for steel antennas and a high-quality system involving several layers of prime and white paint based on zinc dust should be applied. The first coats are usually applied in the workshop; the last coat is applied at site during the erection.

At site the lower part of the receiver cabin must be bolted or welded on the azimuth fork, the upper part of the receiver cabin is then bolted on the lower part. The elevation bearings assembly is mounted on the fork arms and the devices in the receiver cabin (e. g. doors, hatches, and fan) shall be installed. Finally, the cabin shall be protected from atmospheric influences by an external cladding with insulation.

Prior to any lift, the azimuth cable twister shall be mounted completely including cable floor and grouting. Then, the azimuth bearing assembly, the spiral staircase and the azimuth fork (following this order) have to be lifted by means of big cranes on top of the pedestal. Once it is aligned horizontally, it has to be connected to the embedded anchor ring by means of anchor bolts. The central part of the fork platform will be connected to the azimuth bearing through a precisely machined contact surface. This whole is then aligned by means of adjusting screws to the alignment specifications that are usually less than 10" and grouted to the concrete tower head with non-shrinking cement grout. The fastening screws must be loaded with a hydraulic device. The ballast cantilever are then lifted and installed on the fork arms.

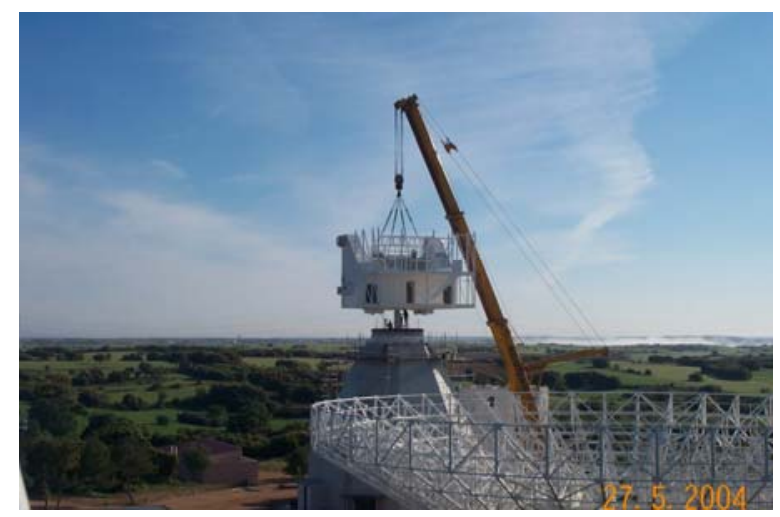

Figure 7: Lifting the fork of the 40 meter IGN radiotelecope. 
The trusswork is assembled on the ground on the concrete foundations specially made for this assembly. The reflector trusswork consisting of main and intermediate trusses, ring girder and ring bracing will arrive at site in individual parts, which have to be welded together. The cladding, fans and electrical cabling should be installed on the ground also. Then the whole has to be lifted and installed on top of the cantilevers and welded to the fork structure. After that, the tetrapod legs with the apex and the subreflector mirror can be bolted to the reflector trusswork.

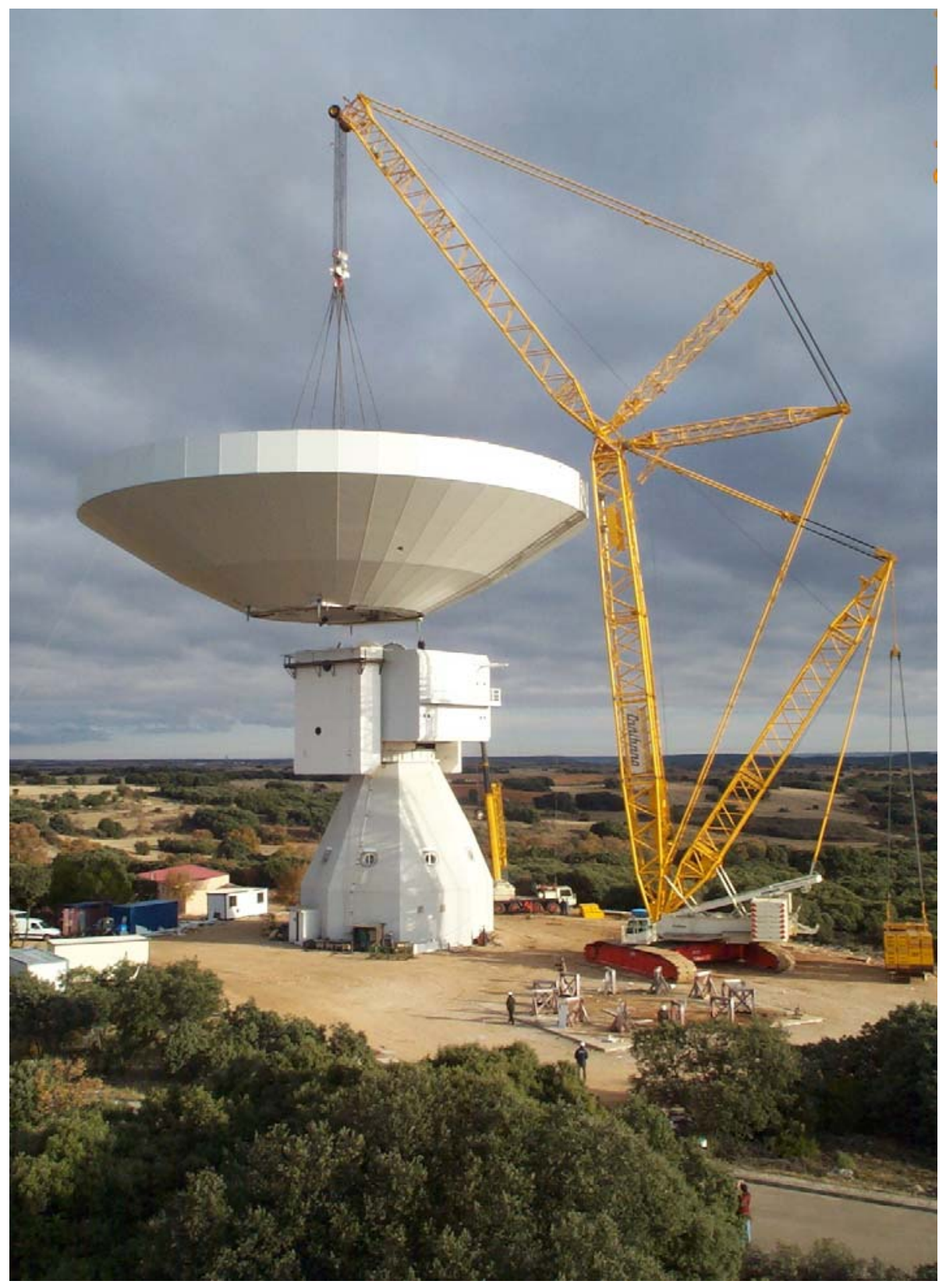

Figure 8: Lifting the main reflector of the 40 meter IGN radiotelescope. 
The drives have to be mounted to the fork. After adjusting of the toothing clearance the gear box supports are pinned up. The gearboxes must be filled with oil, the gear rims have to be greased. The encoder supports, the actuating shafts, the motor driven locking devices, the limit switches and the auxiliary equipment must be mounted and adjusted. Finally, with the installation of the servo racks the antenna can be moved in both axes.

The reflecting panels can be installed. The panels have to be connected to the supporting structure by means of special setting elements. With this supports the panels can be adjusted from the panel top side.

Once the construction phase is finished, the alignments and adjustment have to be done. First the balance can take place by pouring a mix of concrete and steel in the ballast arms. The elevation axis horizontal alignment has to be adjusted with the specified accuracy, normally close to 5”, by means of high precision level.

The reflector panels shall be set to the specified accuracy. The adjustment of the panel surface will be effected in the Elevation $=45^{\circ}$ position. Alignment of panels is made by means of adjusters and it is a tedious task. It is accomplished by first rough aligning the support clips of the backstructure with a tape measure stretched from a center theodolite station and setting them to an elevation angle. Final alignment is done by a worker who turns the adjuster following the instructions of the theodolite operator at the vertex of the parabola. Best fit data reduction of the theodolite angles yields angular changes needed to finish setting the reflector surface to the desired surface accuracy.

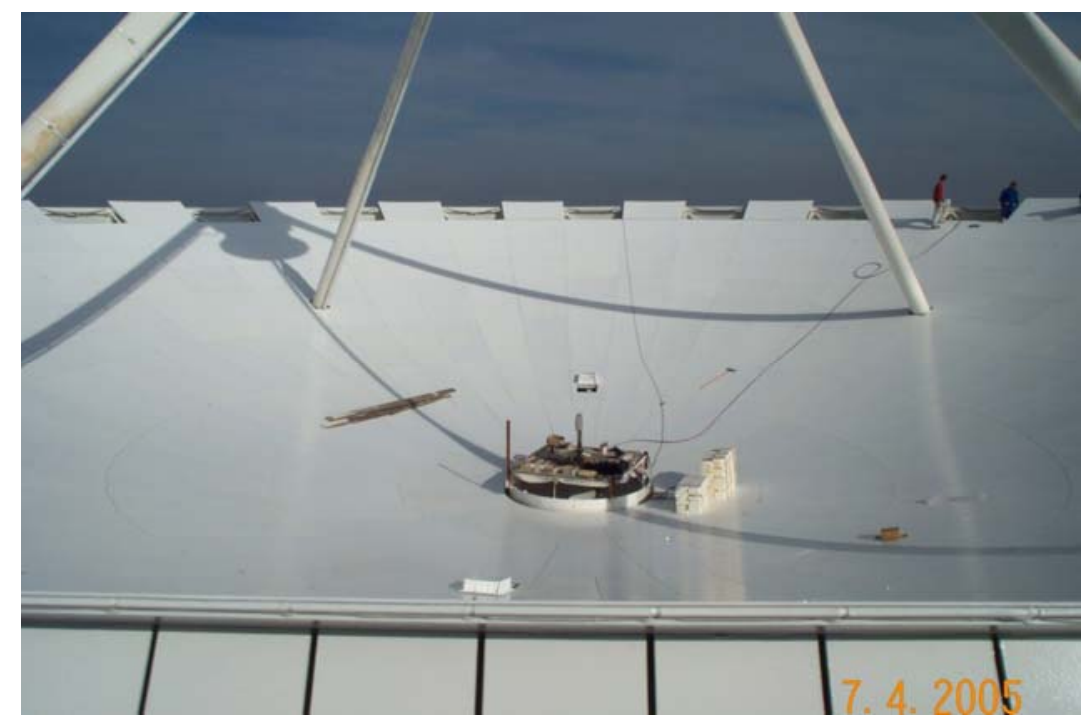

Figure 9: Aligning the main dish panels.

The final alignment is then performed using modern techniques of holography [10]. Microwave holography has proven to be a valuable technique for characterizing the mechanical and RF performance of large reflector antennas. This technique utilizes the Fourier transform relation between the far-field radiation pattern and the aperture-field distribution. Resulting aperture phase and amplitude data can be used to characterize various performance parameters 
including panel alignment, panel shaping, subreflector position, antenna aperture illumination and gravity deformation effects.

The final commissioning of the servo system and the installation of the first receivers can be them accomplished. The first observations should offer a check of the real performance of the radiotelescope.

\section{Big telescopes}

Some of the biggest radiotelescopes around the world:

o Parkes Radiotelescope.

o Australia 1962

o 64 meter diameter

o 13 beam focal plane array

o Resurfaced in 1975

o Lovell Telescope

o UK 1950s

o 76.2 meter diameter

o Fully efficient to $6 \mathrm{GHz}$

o Kitt Peak

o Arizona, USA, 1967

o 12 meter diameter

o Pionnering millimeter wave

o MRT

o Granada, Spain, 1982

o 30 meter diameter

o Fully function mm-wave for 3, 3 and $1.3 \mathrm{~mm}$ bands

o 50 microns accuracy

o JCMT

o Hawaii, USA

- 15 meter diameter

o 25 microns surface accuracy

o SIS receivers until $690 \mathrm{GHz}$

o CST

o Hawaii, USA

o Diameter 10.4 meter

o $665 \mathrm{GHz}$

o HHT

o Arizona, USA

o 10 meter diameter

o 15 microns surface accuracy

o LMT

o Mexico, under construction

o 50 meter diameter

o 4600 meter altitude

o Millimeter range 
o Effelsberg
o Germany
o 100 meter diameter
o 3 milimeter operation

o Green Bank
o West Virginia, USA
o >100 meter diameter
o Dual offset Gregorian
o Active surface
o Until $50 \mathrm{GHz}$

o Nobeyama
o Japan
o 45 meter diameter
o 200 microns surface accuracy

o SRT

o Italy, under construction

o 64 meter diameter

o $300 \mathrm{MHz}$ to $100 \mathrm{GHz}$

o ARIES

o Yebes, Spain, 2005

o 2 to $140 \mathrm{GHz}$

o Arecibo

o Puerto Rico, USA

o 300 meter diameter

o To $10 \mathrm{GHz}$

o Biggest telescope

o FAST

o China, under construction

o 500 meter diameter

o Nançay
o Paris, 1964
o To $3 \mathrm{GHz}$
o Spherical mirror

\section{Arrays}

o Westerbork

o Netherlands

o Centimetre array

o Synthesis radiotelescope

o 14 antennas 25 meter diameter

o $300 \mathrm{MHz}$ to $9 \mathrm{GHz}$

o VLA

o New Mexico, USA

o 27 moveable antennas 25 meter diameter

o $73 \mathrm{MHz}$ to $50 \mathrm{GHz}$ 
O ATCA
o Australia
- Australia telescope compact array
o 6 antennas 22 meter diameter
o 1 to $9 \mathrm{GHz}$
o Being upgraded to 22 and $100 \mathrm{GHz}$

o Ryle Telescope

o Cambridge, UK

- 8 antennas 13 meter diameter

o To $15 \mathrm{GHz}$

o DRAO

o Canada

o 7 antennas 9 meter diameter

o 408 to $1420 \mathrm{MHz}$

o Mauritius radiotelescope

o Mauritius

o 1088 helical antennas

o $151 \mathrm{MHz}$

o GMRT

o India

o Giant Metrewave radio telescope

o 30 fixed antennas 45 meter diameter

o $150-1420 \mathrm{MHz}$

o Plateau de Bure

o France

0 Interferometer

- 6 antennas 15 meter diameter

o 80 to $250 \mathrm{GHz}$

o CMA

o California, SA

o Caltech millimeter array

o 6 antennas 10.4 meter diameter

o $\quad 86-270 \mathrm{GHz}$

o BIMA

o California, USA

o Berkeley-Illinois-Maryland Association

o 10 antennas 6 meter diameter

o 70 to $270 \mathrm{GHz}$

O NMA

o Japan

o Nobeyama Millimeter Array

o 6 antennas 10 meter diameter

o $\quad 85$ to $237 \mathrm{GHz}$

o SMA

o Hawaii, USA

o Submillimeter Array

o 8 antennas 6 meter in diameter

o 190 to $850 \mathrm{GHz}$

o ATCA

o The only southern Millimetre interferometer

o $\quad 3-12$ and 7 millimeter. 
O ALMA

o Chile

o Atacama Large Millimetre Array

o SKA

o www.alma.cl

o Square Killometer Array

\section{References}

[1] P. W. Hannan, Microwave Antennas Derived from the Cassegrain Telescope, IRE Trans. Vol. PGAP-9, March 1961

[2] GRASP 9, Ticra Enginnering Consultants, 2004, http://www.ticra.com

[3] Paul F. Goldsmith, Quasioptical Systems, IEEE Press, 1998

[4] A.D. Olver, Microwave Horns and Feeds, IEEE Press, 1994

[5] ANSYS CFD Technologies featuring., http://www.ansys.com

[6] S. Hoerner, Homologous deformation of tiltable telescopes, J. Structural Div. Proc. Amer. Soc. Civil Engineers, Vol. 93, No. ST5, October 1967

[7] Takashi Kitsuregawa, Advanced Technology in Satellite Communication antennas, Artech House, 1990

[8] IRAM, Institute de Radioastronomie Millimetrique, Grenoble (France), http://www.iram.fr

[9] ALMA, Large Atacama Millimeter Array, http://www.alma.nrao.edu

[10] David J. Rochblatt, Boris L. Seidel, Microwave Antenna Holography, IEEE Transactions on Microwave Theory and Techniques, Vol. 40, No. 6, 1992 